\title{
Von der Rechtsgemeinschaft zur Verweigerungsunion?
}

\author{
Von Nina Wunderlich, Berlin*
}

Der vorliegende Aufsatz widmet sich der Frage, ob sich die Europäische Rechtsgemeinschaft aufgrund einer etwaigen Verweigerungshaltung der EU-Mitgliedstaaten in einer Krise befindet, wie kritische Stimmen es zum Teil nahe legen. Anhand einer allgemeinen Bestandsaufnahme zu den Vertragsverletzungsverfahren, die die Europäische Kommission in den letzten Jahren eingeleitet hat, sowie einer Betrachtung mitgliedstaatlichen Handelns im Rahmen der Finanz- und Wirtschaftskrise sowie der Migrationskrise, nähert sich der Beitrag der Frage, ob sich die Mitgliedstaaten im Grundsatz rechtstreu verhalten. Gegenstand der Untersuchung ist dabei auch, ob Verstöße gegen das in Art. 2 EUV verankerte Rechtsstaatsprinzip durch Vertragsverletzungsverfahren - ggf. neben Verfahren nach Art. 7 EUV-geahndet werden können.

Die Frage, ob sich die europäische Rechtsgemeinschaft in der Krise befindet, wird in den letzten Jahren in der Literatur verstärkt diskutiert. Diese Diskussion knüpfte zunächst vor allem am Umgang der EU-Institutionen und der Mitgliedstaaten mit den Herausforderungen der Finanz- und Wirtschaftskrise an und setzte sich insbesondere im Hinblick auf die Migrationskrise - etwa die Reaktion Ungarns auf das Urteil des Europäischen Gerichtshofs vom 6. September 2017 zur Frage der Flüchtlingsverteilung - fort. Die Diskussion um die Situation der Rechtsgemeinschaft reicht aber über diese Bereiche hinaus, indem den Mitgliedstaaten in eher allgemeiner Form vorgehalten wird, im Zweifel nationale Eigeninteressen vor die Erfüllung rechtlicher Pflichten zu stellen.

In diese Richtung wiesen etwa einige Pressestimmen, die eine Krise der Rechtsgemeinschaft anmahnten ${ }^{1}$ oder in denen gar die Rede davon war, dass sich die Rechtsgemeinschaft Walter Hallsteins zu einer „Rechtsbruchgemeinschaft“ entwickelt habe. ${ }^{2}$ Es wurde in diesem Zusammenhang etwa ausgeführt: „Wer gegen zentrale Bestimmungen verstößt, muss kaum mit Sanktionen rechnen. Entweder drücken die Kontrolleure alle Augen zu, oder man leitet derart langwierige und beeinflussbare Verfahren ein, dass kein Land sie ernsthaft fürchtet. “3

* Ministerialrätin Dr. Nina Wunderlich, LL.M., leitet das Zentrale Rechtsreferat im Bundesministerium für Wirtschaft und Energie. Die Verfasserin gibt in diesem Beitrag ausschließlich ihre persönliche Ansicht wieder. Der vorliegende Beitrag basiert auf einem Vortrag, den die Verfasserin am 15. September 2017 bei der 36. Tagung der Gesellschaft für Rechtsvergleichung in Basel gehalten hat; er wurde im Hinblick auf die weiteren Entwicklungen seitdem überarbeitet und aktualisiert.

1 R. Scholz, EU: Die marode Rechtsgemeinschaft, 27.10.2016, www.theeuropean.de/rupert-scholz-scholz/11442europäische-union-in-der-krise.html.

2 C. Schlitz, Das sind die vier Gründe für die Krise der EU, 22.6.2017, https://www.welt.de/politik/ausland/articl e156373989/Das-sind-die-vier-Gruende-fuer-die-Krise-der-EU.html.

3 C. Schlitz, a.a.O.; der auch darauf hinweist, dass Deutschland regelmäßiger Adressat von Vertragsverletzungsverfahren ist, $C$. Schlitz, Die ausgezehrte EU dümpelt weiter vor sich hin, https://www.welt.de/debatte/kommen tare/article156685649/Die-ausgezehrte-EU-duempelt-weiter-vor-sich-hin.html. 
Der Schwerpunkt der nachfolgenden Betrachtungen wird auf dem Handeln der Mitgliedstaaten liegen. Missachten sie tatsächlich Unionsrecht? Und wenn ja: aus welchen Gründen? Zur Beantwortung dieser Fragen soll der Versuch einer Bestandsaufnahme der für die Diskussion um den Stand der Rechtsgemeinschaft aus Sicht der Verfasserin wesentlichen Bereiche unternommen werden. Eine solche Bestandsaufnahme kann - da jeder der zu behandelnden Bereiche für sich genommen Gegenstand eingehender Untersuchungen sein könnte bzw. bereits heute ist naturgemäß nur sehr kursorisch sein und muss sich auf einige ausgewählte Punkte beschränken.

Der vorliegende Beitrag verzichtet bewusst darauf, den Versuch einer Definition des Begriffs der Rechtsgemeinschaft voranzustellen, dessen Grundlage Walter Hallstein in den 1960er Jahren gelegt hat. ${ }^{4}$ Das heute möglicherweise grundsätzlich geänderte Verständnis des Begriffs einer Rechtsgemeinschaft im Hinblick auf die europäische Integration wurde bereits ausführlich analysiert. ${ }^{5}$ Der vorliegende Beitrag will sich der Frage, ob mitgliedstaatliches Handeln im Begriff ist, den Boden einer Rechtsgemeinschaft $\mathrm{zu}$ verlassen, vielmehr anhand von Fallbeispielen nähern. Bestimmte Kriterien, die aus Sicht der Verfasserin für das Fortbestehen der Europäischen Union als Rechtsgemeinschaft essentiell sind, werden sich bei der Betrachtung der Fallbeispiele herauskristallisieren.

Um den Grad der Befolgung des EU-Rechts durch die Mitgliedstaaten aus eher übergreifender Perspektive beurteilen zu können, widmet sich die folgende Untersuchung zunächst einer allgemeinen Bestandsaufnahme zu den Vertragsverletzungsverfahren, die die Europäische Kommission gegen die EU-Mitgliedstaaten eingeleitet hat (I.). Im Hinblick darauf, dass mitgliedstaatliches Handeln insbesondere im Rahmen der Finanz- und Wirtschaftskrise sowie in Bezug auf die Behandlung der Flüchtlingskrise vielfach dem Vorwurf des Rechtsbruchs ausgesetzt war, sollen diese beiden Bereiche näher untersucht werden (II. und III.). Schließlich widmet sich der Beitrag gerade in Bezug auf die gegen Polen und Ungarn eingeleiteten Rechtsstaatsverfahren nach Art. 7 EUV sowie die parallel geführten Vertragsverletzungsverfahren zu gleichgelagerten Rechtsverstößen der Frage, was Vertragsverletzungsverfahren in diesem Bereich leisten können und wo ihre Grenzen liegen (IV.).

4 Vgl. W. Hallstein, Die EWG - Eine Rechtsgemeinschaft, Rede gehalten anlässlich der Ehrenpromotion an der Universität Padua am 12. März 1962, in: Oppermann (Hrsg.), Hallstein - Europäische Reden, 1979, S. 341 ff.; ders., Die Europäische Gemeinschaft, 5. Aufl. 1979, S. 51 ff.

5 Vgl. z.B. A. v. Bogdandy, Jenseits der Rechtsgemeinschaft - Begriffsarbeit in der europäischen Sinn- und Rechtsstaatlichkeitskrise, EuR 2017, S. 487 ff. Siehe zum Begriff der Rechtsgemeinschaft auch M. Nettesheim, „Gegründet auf Werten...": Das Narrativ der Wertegemeinschaft und der Sanktionsmechanismus des Art. 7 EUV, in: Franzius/Mayer/Neyer (Hrsg.), Die Neuerfindung Europas - Bedeutung und Gehalte von Narrativen für die europäische Integration, 2019, S. 91, $99 \mathrm{ff}$. 


\section{Vertragsverletzungsverfahren allgemein}

Bei einer Bestandsaufnahme zur Frage, ob die Mitgliedstaaten sich noch innerhalb der Grenzen einer Rechtsgemeinschaft bewegen, bietet es sich an, zunächst allgemein bei den Vertragsverletzungsverfahren der Europäischen Kommission gegen Mitgliedstaaten der EU und deren Statistik anzusetzen. Denn dies gibt zumindest einen ersten Anhaltspunkt für die Frage des Stands der Befolgung von Unionsrecht durch die Mitgliedstaaten.

Hinsichtlich der Anzahl der jeweils laufenden Vertragsverletzungsverfahren ist festzustellen, dass in den letzten Jahren die Zahl der anhängigen Vertragsverletzungsverfahren gegen alle Mitgliedstaaten insgesamt gestiegen ist. Zwar ist, wie sich aus dem Jahresbericht der Europäischen Kommission für das Jahr 2017 ergibt, die Zahl der Ende 2017 insgesamt anhängigen Verfahren mit 1559 gegenüber 2016, in denen es noch 1657 waren, ${ }^{6}$ leicht gesunken. ${ }^{7}$ Diese Entspannung in 2017 dürfte jedoch insbesondere darauf zurückzuführen sein, dass die Kommission - wie sie in ihrer Mitteilung „EU-Recht: Bessere Ergebnisse durch bessere Anwendung" vom Dezember 2016 dargelegt hat - einen neuen Ansatz bei der Verfolgung von potentiellen Rechtsverstößen durch die Mitgliedstaaten praktiziert. ${ }^{8}$ Neben einer stärkeren Zusammenarbeit mit den Mitgliedstaaten bei der Durchsetzung von EU-Recht will die Kommission das ihr zustehende Ermessen bei Vertragsverletzungsverfahren im Sinne einer stärkeren Prioritätensetzung dahingehend ausüben, dass sie in erster Linie die schwerwiegenden Verstöße gegen EURecht verfolgt, die die Interessen der Bürgerinnen und Bürger und der Wirtschaft beeinträchtigen. ${ }^{9}$ Vor dem Hintergrund dieser - insgesamt auch im Hinblick auf den Subsidiaritätsgrundsatz nach Art. 5 EUV sehr zu begrüßenden - geänderten Praxis der Kommission dürfte aus dem Rückgang der Verfahren in 2017 noch keine grundsätzliche Umkehr der Gesamttendenz eines Anstiegs der Vertragsverletzungsverfahren in den letzten Jahren resultieren. Denn die insgesamt anhängigen Verfahren lagen in 2012 noch bei $1343^{10}$. Vor allem zwischen den Jahren 2015 und 2016 hat es einen besonders deutlichen Anstieg der Vertragsverletzungsverfahren um etwa $20 \%$ gegeben. ${ }^{11}$ Die Politikbereiche, in denen der Hauptteil der

6 Jahresbericht der Kommission 2016, Kontrolle der Anwendung des EU-Rechts vom 6.7.2017, KOM (2017) 370 final, S. 30.

7 Jahresbericht der Kommission 2017, Kontrolle der Anwendung des EU-Rechts vom 12.7.2018, KOM (2018) 540. Ende 2018 waren 1571 Verfahren anhängig (ein Anstieg von 0,8\% gegenüber 2017), s. Jahresbericht der Kommission 2018, Kontrolle der Anwendung des EU-Rechts vom 4.7.2019, KOM (2019) 319, Part I, General statistical overview, S. 19.

8 Europäische Kommission, Mitteilung vom 21.12.2016, EU-Recht: Bessere Ergebnisse durch bessere Anwendung (2017/C18/02), AB1. EU C 18/10.

9 Europäische Kommission, Mitteilung vom 21.12.2016, EU-Recht: Bessere Ergebnisse durch bessere Anwendung (2017/C18/02), ABl. EU C 18/10, 14.

10 Jahresbericht der Kommission 2012 über die Kontrolle der Anwendung des EU-Rechts vom 22.10.2013, KOM (2013) 726 final, S. 9.

11 Kommission sieht hierin Anlass zur Sorge; Pressebericht der Kommission vom 6.7.2017 „Mitgliedstaaten setzen EU-Recht nicht ausreichend um, https://ec.europa.eu/germany/news/bericht-mitgliedstaaten.setzen-eu-rec ht-nicht-ausreichend-um.de. 
Vertragsverletzungsverfahren eingeleitet wurden, waren dabei in 2016 die Bereiche des Binnenmarktes, der Umwelt sowie des Finanzsektors. ${ }^{12}$ Diese Verteilung auf die hauptsächlich betroffenen Politikbereiche entspricht im Großen und Ganzen auch der der Vorjahre - in 2014 und 2015 gehörte allerdings auch der Verkehrssektor zu den am stärksten betroffenen Bereichen. ${ }^{13}$

Gestiegen ist in den letzten Jahren nicht nur die Gesamtzahl der gegen die Mitgliedstaaten eingeleiteten Vertragsverletzungsverfahren, sondern auch der Anteil der Verfahren wegen der verspäteten Umsetzung von Richtlinien. Diese Verfahren sind seit 2012 nicht nur stetig angewachsen - von 447 in 2012 auf 847 Verfahren in 2016. Vielmehr ist auch hier von 2015 auf 2016 ein besonders starker Anstieg zu verzeichnen: Von 543 Vertragsverletzungsverfahren in 2015 bei insgesamt 56 umzusetzenden Richtlinien auf 847 in 2016 bei 70 umzusetzenden Richtlinien. ${ }^{14}$ In 2017 ist die Anzahl dieser Verfahren mit 808 jedoch wieder leicht zurückgegangen. ${ }^{15}$

Auch die Zahl der gegen Deutschland eingeleiteten Vertragsverletzungsverfahren ist seit 2012 deutlich angestiegen, während zuvor die Anzahl der Verfahren noch in der Tendenz rückläufig gewesen war: So waren am 31.12.2016 gegen Deutschland insgesamt 91 Vertragsverletzungsverfahren anhängig. Diese Zahl ist auch im Vergleich zu anderen Mitgliedstaaten hoch; nur gegen Spanien liefen zu diesem Stichtag genauso viele Vertragsverletzungsverfahren. ${ }^{16}$ Allerdings bewegt sich Deutschland mit 29 in 2016 neu eröffneten Vertragsverletzungsverfahren im unteren Bereich der 28 Mitgliedstaaten ${ }^{17}$ Auch bei der Umsetzung von Richtlinien kann Deutschland auf eine deutlich positivere Bilanz blicken: Im Jahr 2016 wur-

12 Jahresbericht der Kommission 2016, Kontrolle der Anwendung des EU-Rechts, vom 6.7.2017, KOM (2017) 370 final, S. 31.

13 Jahresbericht der Kommission 2014, Kontrolle der Anwendung des EU-Rechts vom 9.7.2015, KOM (2015) 329 final, S. 15; Jahresbericht der Kommission 2015, Kontrolle der Anwendung des EU-Rechts vom 15.7.2016, KOM (2016) 463 final, S. 25. So auch in 2018, s. Jahresbericht der Kommission 2018, Kontrolle der Anwendung des EU-Rechts vom 4.7.2019, KOM (2019) 319, Part I, General statistical overview, S. 20.

14 Jahresbericht der Kommission 2016, Kontrolle der Anwendung des EU-Rechts, vom 6.7.2017, KOM(2017) 370 final, S. 33.

15 Siehe Statistischen Annex zum Jahresbericht der Kommission 2017, SWD (2018)377, S. 15. Diese Entwicklung ist bemerkenswert im Hinblick auf den durch den Vertrag von Lissabon neu eingeführten Art. 260 Abs. 3 AEUV, der bei Vertragsverletzungsverfahren wegen Nichtumsetzung einer Richtlinie eine beschleunigte Verhängung von finanziellen Sanktionen vorsieht. Die Kommission hat von dieser Möglichkeit umfassend Gebrauch gemacht, jedoch zunächst im Regelfall allein die Verhängung von Zwangsgeldern - und nicht von Pauschalbeträgen - beantragt. Dies hatte im Ergebnis dazu geführt, dass die Mitgliedstaaten noch im laufenden Gerichtsverfahren die Richtlinie umsetzen konnten - mit der Folge, dass sich der Klagegegenstand vor Urteilsverkündung erledigte und die Kommission die Klagen zurückzog. Angesichts der trotz Anwendung des Art. 260 Abs. 3 AEUV steigenden Zahlen von Vertragsverletzungsverfahren hat die Kommission in ihrer Mitteilung ,EU-Recht: Bessere Ergebnisse durch bessere Anwendung“" vom 19. Januar 2017 angekündigt, dass sie künftig neben Zwangsgeldern auch die Verhängung eines Pauschalbetrags beantragen wird (Mitteilung der Kommission „EU-Recht: Bessere Ergebnisse durch bessere Anwendung“ (2017/C18/02), AB1. EU C 18/10, 15). Damit erledigt sich bei einer Umsetzung der Richtlinie im Klageverfahren die Klage nicht mehr; denn es entfällt für den Gerichtshof nur die Notwendigkeit, über den Zwangsgeldantrag zu entscheiden, nicht aber die Erforderlichkeit, einen Pauschalbetrag als Sanktion für das vorausgehende Fehlverhalten festzusetzen.

16 Jahresbericht der Kommission 2016, Kontrolle der Anwendung des EU-Rechts, vom 6.7.2017, KOM (2017) 370 final, S. 30.

17 Jahresbericht der Kommission 2016, Kontrolle der Anwendung des EU-Rechts, vom 6.7.2017, KOM (2017) 370 final, S. 28. 
den gegen Deutschland 21 Verfahren wegen einer verspäteten Umsetzung eingeleitet $^{18}$; in 2017 waren es $19^{19}$, in 201813 Verfahren $^{20}$. Für einen großen Mitgliedstaat, der aufgrund seiner föderalen Struktur bei der Umsetzung von Richtlinien besonderen Herausforderungen begegnet, erscheint dies als ein sehr akzeptables Ergebnis.

Die insgesamt gestiegenen Zahlen bei den Vertragsverletzungsverfahren werfen zwar auf den ersten Blick kein gutes Licht auf die Befolgung der EU-Rechtsnormen durch die Mitgliedstaaten. Die Gründe für eine Nichtbefolgung europäischen Rechts liegen aber in aller Regel nicht in einer „Verweigerungshaltung“, sondern haben vielfältige Ursachen. Im Falle der Nichtumsetzung von Richtlinien geht es beispielsweise häufig um Verspätungen von einigen Monaten, die durch innerstaatliche Strukturen - etwa bei Föderalstaaten - politische Blockaden in den jeweiligen nationalen Parlamenten oder weitere Schwierigkeiten des jeweiligen Mitgliedstaats bedingt sind. Selbstverständlich sind solche internen Belange nach ständiger Rechtsprechung des Gerichtshofs für die Frage des Bestehens einer Vertragsverletzung unerheblich. ${ }^{21}$ Dennoch lässt sich in solchen Fällen nicht von einer Missachtung des Rechts sprechen.

Hinzuzufügen ist, dass die genannten Zahlen allein nicht aussagekräftig sind, wenn es um eine Annäherung an die Beantwortung der Frage geht, ob die Mitgliedstaaten sich im Ergebnis rechtstreu verhalten. Vielmehr ist zur Beantwortung dieser Frage zunächst auch der weitere Verlauf der Vertragsverletzungsverfahren zu betrachten. Dabei ist insbesondere zu beachten, ob und in welchem Maße die Mitgliedstaaten bereit sind, dem von der Kommission geäußerten Rechtsverletzungsvorwurf, soweit er tatsächlich berechtigt ist, im Laufe des Verfahrens - vor einer Entscheidung durch den Gerichtshof - abzuhelfen. Hierzu führte die Kommission in ihrem Jahresbericht 2016 aus, dass die „Statistiken zeigen, dass die Mitgliedstaaten sehr bemüht sind, ihre Verstöße abzustellen, bevor der Gerichtshof eine Entscheidung fällt" ${ }^{22}$ Entsprechend konnte die Kommission in 2016 einen großen Teil der Fälle vor einem Urteil des Gerichtshofs abschließen. ${ }^{23}$ Auch Deutschland ist es in den letzten Jahren durchgehend gelungen, einen Großteil der Vertragsverletzungsverfahren noch im Vorverfahren zu bereinigen - sei es durch Behebung eines tatsächlich vorliegenden Rechtsverstoßes bzw. der schnellstmög-

18 Jahresbericht der Kommission 2016, Kontrolle der Anwendung des EU-Rechts, vom 6.7.2017, KOM (2017) 370 final, S. 34.

19 Siehe Statistischen Annex zum Jahresbericht der Kommission 2017, SWD (2018) 377, S. 16.

20 Jahresbericht der Kommission 2018, Kontrolle der Anwendung des EU-Rechts vom 4.7.2019, KOM (2019) 319, Part I, General statistical overview, S. 26.

21 Siehe nur z.B. EuGH, Urt. v. 12.12.1996, Rs. C-297/95 (Kommission/Deutschland), ECLI:EU:C:1996:500, Slg. 1996, I-06739, Rn 8 f (st Rspr.). Siehe auch z.B. H.-J. Cremer, in: Calliess/Ruffert, EUV/AEUV, 5. Aufl. 2016, Art. 258 AEUV, Rn. 34 mwN; N. Wunderlich, in: von der Groeben/Schwarze/Hatje, 7. Aufl. 2015, Rn. 43 mwN.

22 Jahresbericht der Kommission 2016, Kontrolle der Anwendung des EU-Rechts, vom 6.7.2017, KOM (2017) 370 final, S. 31.

23 Jahresbericht der Kommission 2016, Kontrolle der Anwendung des EU-Rechts, vom 6.7.2017, KOM (2017) 370 final, S. 31. 
lichen Nachholung einer verspäteten Richtlinienumsetzung, sei es durch Überzeugung der Kommission, dass ein EU-Rechtsverstoß entgegen ihrer ursprünglichen Auffassung nicht vorliegt. So lagen Ende 2015 von insgesamt 89 Vertragsverletzungsverfahren nur 4 Klagebeschlüsse sowie 5 beim EuGH anhängige Vertragsverletzungsklagen vor, in 2016 war von insgesamt 91 Verfahren in 4 Verfahren von der Kommission Klageerhebung beschlossen worden und in weiteren 7 Verfahren war eine Klage beim EuGH anhängig. Ende des Jahres 2017 waren gegen Deutschland 74 Vertragsverletzungsverfahren anhängig, davon 5 beim EuGH.

Es ist ferner hervorzuheben, dass die bloße Tatsache der Einleitung eines Vertragsverletzungsverfahrens durch die Kommission keinesfalls zwingend mit einem tatsächlich vorliegenden Rechtsverstoß gleichzusetzen ist. Hat es ein Mitgliedstaat versäumt eine Richtlinie umzusetzen, dürfte zwar grundsätzlich keine gerichtliche Klärung dieser Tatsache erforderlich sein; aus diesem Grund sieht Art. 260 Abs. 3 AEUV die Möglichkeit eines verkürzten Verfahrens bis zur Verhängung finanzieller Sanktionen vor. In allen Fällen jedoch, in denen die Rechtslage nicht klar ist und ein rechtliches Fehlverhalten gerade von dem betroffenen Mitgliedstaat bestritten wird, kann erst mit dem Urteil des Gerichtshofs verbindlich feststehen, ob und ggf. inwieweit ein Verstoß gegen EU-Recht tatsächlich vorliegt. Auch wenn die Kommission in einer überwiegenden Zahl der Verfahren vor dem Gerichtshof in Vertragsverletzungsverfahren obsiegt, hat der Gerichtshof doch auch in einigen Vertragsverletzungsurteilen letztlich den Mitgliedstaaten auch Deutschland ${ }^{24}$ - Recht gegeben.

Dies zeigt, was ohnehin seit Anbeginn der europäischen Integration inhärenter Bestandteil der EU als Rechtsgemeinschaft ist: das Ringen um das Recht, um die Auslegung der jeweiligen Rechtsnormen. Solange die Mitgliedstaaten insoweit ihre rechtlichen Standpunkte bis vor den Gerichtshof verteidigen, kann im Regelfall nicht von einer „Missachtung“ bzw. „Verweigerung“ ausgegangen werden. Wie die obenstehenden Ausführungen gezeigt haben, lassen sich daher auch aus der reinen Zahl der Vertragsverletzungsverfahren keine unmittelbaren Schlussfolgerungen in Bezug auf den tatsächlichen Grad der Rechtsbefolgung durch einen Mitgliedstaat ziehen.

Essentiell in einer Rechtsgemeinschaft ist dann jedoch die Akzeptanz eines verbindlichen Urteils des Gerichtshofs, auch wenn der betroffene Mitgliedstaat im Einzelfall nicht einverstanden damit sein mag. Wann es an dieser Akzeptanz fehlt und damit eine echte Missachtung eines solchen Urteils durch einen Mitgliedstaat vorliegt, die den Prinzipien einer Rechtsgemeinschaft entgegenläuft, muss im Einzelfall betrachtet werden. Jedenfalls dürfte sich ein solch fundamentaler Vor- 
wurf nicht allein daran knüpfen, dass ein Urteil nicht, nicht vollständig oder erst nach langem Zeitablauf umgesetzt wurde. Auch hierfür mögen besondere, auch rechtliche Gründe vorliegen, die der Mitgliedstaat im Laufe eines Zwangsgeldverfahrens nach Art. 260 Abs. 2 AEUV vorbringen kann. ${ }^{25}$

Eine blanke Missachtung einer Entscheidung des Gerichtshofs ohne rechtliche Rechtfertigung wäre dagegen bedenklich. Das fortgesetzte Abholzen von Bäumen des polnischen Bialowieska-Urwalds durch die polnischen Behörden deutete zunächst dahin, dass die polnische Regierung eine Entscheidung des Gerichtshofs offen missachten wolle. ${ }^{26}$ Dem begegneten Kommission und letztlich der Gerichtshof jedoch im Ergebnis erfolgreich. In dem gegen Polen zu diesem Vorwurf initiierten Vertragsverletzungsverfahren erließ der Gerichtshof (Große Kammer) am 20. November 2017 einen Beschluss im vorläufigen Rechtsschutz, der der Republik Polen umgehend bis zur Verkündung des Urteils in der Hauptsache aufgab, die Maßnahmen der aktiven Waldbewirtschaftung in den betroffenen Gebieten des Urwalds, die Beseitigung von über hundert Jahre alten Fichten und das Fällen von Bäumen im Rahmen der Holzgewinnung im Bialowieska-Urwald, einzustellen. Eine Ausnahme gewährte der Gerichtshof nur für unbedingt erforderliche Maßnahmen zur Gewährleistung der öffentlichen Sicherheit von Personen in unmittelbarer Umgebung der Verkehrswege oder sonstiger bedeutsamer Infrastrukturen. Besonders hervorzuheben ist bei diesem Beschluss des Gerichtshofs im vorläufigen Rechtsschutz eines Vertragsverletzungsverfahrens, dass der Gerichtshof bereits in diesem Stadium die Auferlegung eines Zwangsgelds gegen Polen in Höhe von mindestens 100.000 Euro pro Tag verbindlich androhte - beginnend mit dem Tag der Bekanntgabe des Beschlusses und so lange, bis Polen diesen Beschluss befolgen würde bzw. bis zur Verkündung des abschließenden Urteils in der Rechtssache C-441/17. ${ }^{27}$ Zudem verpflichtete er die polnische Regierung, der Kommission spätestens 15 Tage nach Bekanntgabe des Beschlusses detailliert sämtliche Maßnahmen darzulegen, die sie ergriffen hat, um dem Beschluss in vollem Umfang nachzukommen.

In seinem Urteil vom 17. April 2018 in der Hauptsache gab der Gerichtshof schließlich der Klage der Kommission in vollem Umfang statt. Der polnische Umweltminister Henryk Kowalczyk versicherte, dass seine Regierung sich an das

25 Siehe etwa im Falle des VW-Gesetzes und zur Frage, ob die Bundesrepublik Deutschland das Urteil des EuGH v. 23.10.2007, Rs. C-112/05 (Kommission/Deutschland), ECLI:EU:C:2007:623, Slg. 2007, I-08995, korrekt umgesetzt hatte: EuGH, Urt. v. 22.10.2013, Rs. C-95/12 (Kommission/Deutschland), ECLI:EU:C:2013:676 (Bestätigung der deutschen Umsetzung).

26 Vgl. Spiegel-Online vom 3.8.2017, Streit um Urwald-Abholzung - Polen sägt an Europas Rechtssystem, www.spiegel.de/politik/ausland/bialowieza-urwald-und-eurgh-streit-zwischen-eu-und-polen.

27 EuGH, Beschl. v. 20.11.2017, C-441/17 R (Kommission/Polen), ECLI:EU:C:2017:877, Rn. 118. Siehe auch schon den vorläufigen Beschluss des Vizepräsidenten des Gerichtshofs vom 27.7.2017, C-441/17 R (Kommission/Polen), ECLI:EU:C:2017:622 „La République de Pologne sauf en cas de menace pour la sécurité publique, cesse, immédiatement et jusqu'au prononcé de l'ordonnance qui mettra fin à la présente procédure de réferé, [...] l'enlèvement d'épicéas centenaires morts ainsi que l'abattage d'arbres dans le cadre de l'augmentation du volume de bois exploitable sur le site PLC200004 Puszcza Bialowieska (Pologna), [...]“. 
Urteil halten werde. Zu Strafzahlungen kam es letztlich nicht, da die unionsrechtswidrige Abholzung mittlerweile beendet worden war. ${ }^{28}$

\section{Mitgliedstaatliche Maßnahmen im Rahmen der Finanzkrise}

Im Zusammenhang mit Unterstützungsmaßnahmen im Rahmen der Finanzkrise ist vielfach der Vorwurf erhoben worden, dass EU-Recht missachtet wurde. Dies gilt für mitgliedstaatliche Unterstützungsmaßnahmen ebenso wie für Maßnahmen der EU als auch der $\mathrm{EZB}^{29}$.

In Bezug auf mitgliedstaatliche Unterstützungsmaßnahmen für Griechenland, aber später auch in Bezug auf weitere Euro-Staaten, wie etwa Irland und Portugal, wird insbesondere gerügt, dass das Bail-out-Verbot gemäß Art. 125 AEUV missachtet worden sei. ${ }^{30}$ Dieser Vorwurf bezog sich zunächst insbesondere auf bilaterale Kredite, die etwa im Rahmen des ersten Griechenland-Hilfspakets im Mai 2010 in Zusammenhang mit Hilfen des Internationalen Währungsfonds in Aussicht gestellt wurden. Er wurde aber auch in Bezug auf die Aktivierung der sog. European Financial Stability Facility sowie deren Nachfolge, den sog. Europäischen Stabilitätsmechanismus (ESM) formuliert, in deren Rahmen aufgrund von Haftungszusagen der beteiligten Mitgliedstaaten und eines hieraus folgenden besonders guten Ratings Kredite zu günstigen Konditionen an notleidende EuroStaaten weitergereicht werden konnten. ${ }^{31}$ Für die Etablierung des ESM wurde der Vertrag über die Arbeitsweise der Europäischen Union auf Beschluss des Europäischen Rates vom 25. März $2011^{32}$ um eine neue Bestimmung - Art. 136 Abs. 3 AEUV - ergänzt. Dieser gestattet den Euro-Mitgliedstaaten ,einen Stabilitätsmechanismus ein[zu]richten, der aktiviert wird, wenn dies unabdingbar ist, um die Stabilität des Euro-Währungsgebiets insgesamt zu wahren." Zudem legt Art. 136 Abs. 3 AEUV fest, dass „,die Gewährung aller erforderlichen Finanzhilfen im Rahmen des Mechanismus [...] strengen Auflagen unterliegen [wird]“.

Der Europäische Gerichtshof bestätigte auf Vorlage des irischen Supreme Court in der Rechtssache Pringle in seinem am 27. November 2012 im Plenum gefassten Urteil die Gültigkeit der oben zitierten Vertragsänderung sowie die Vereinbar-

28 Vgl. hierzu z.B. Zeit Online v. 17.4.2018, Abholzung im Bialowieza-Urwald verstößt gegen EU-Recht, www.zeit.de/wissen/umwelt/2018-04/polen-abholzung-in-urwald-verstoesst-gegen-eu-recht.

29 Siehe zur Frage einer möglichen Kompetenzüberschreitung der EZB bei Auflage des Anleihekaufprogramms PSPP und dessen Vereinbarkeit mit dem Verbot der monetären Staatsfinanzierung gem. Art. 123 AEUV, EuGH, Urteil v. 11.12.2018, C-493/17 (Weiss u.a.), ECLI:EU:C:2018:1000 auf Vorlage des BVerfG vom 18.7.2017.

30 H. Kube/E. Reimer, Grenzen des Europäischen Stabilitätsmechanismus, NJW 2010, S. 1911; M. Ruffert, Europäische Währungspolitik als Herausforderung für das Recht, Zentrum für Europäisches Wirtschaftsrecht, Vorträge und Berichte Nr. 214, m.w.N. Siehe hierzu auch z.B. C. Calliess, Das europäische Solidaritätsprinzip und die Krise des Euro - Von der Rechtsgemeinschaft zur Solidaritätsgemeinschaft?, Vortrag an der Humboldt-Universität zu Berlin am 18.1.2011, S. 10 f.

31 H. Kube/E. Reimer, Grenzen des Europäischen Stabilitätsmechanismus, NJW 2010, S. 1911, 1913.

32 Beschluss 2011/199/EU des Europäischen Rates vom 25.3.2011 zur Änderung des Art. 136 des Vertrages über die Arbeitsweise der Europäischen Union hinsichtlich eines Stabilitätsmechanismus für die Mitgliedstaaten, deren Währung der Euro ist (ABl. EG 2011 L 91/1). 
keit der Errichtung des ESM mit den Verträgen. ${ }^{33}$ Der Gerichtshof führte dabei aus, dass Art. 125 AEUV nicht jede Form finanzieller Unterstützung eines anderen Mitgliedstaates ausschließe, soweit der Hauptzweck dieser Vorschrift - sicherzustellen, dass die Mitgliedstaaten auf eine solide Haushaltspolitik achten gewahrt bleibe. ${ }^{34}$ Er hob hervor, dass der ESM selbst keine Bürgschaft für die Schulden des Empfängermitgliedstaates stelle, sondern der Empfängermitgliedstaat gegenüber seinen Gläubigern für seine finanzielle Verbindlichkeiten haftbar bleibt - und gegenüber dem ESM zur Rückzahlung der gewährten Finanzhilfe einschließlich Zinsen verpflichtet ist. Auch in Bezug auf den Ankauf von Anleihen eines ESM-Mitglieds am Sekundärmarkt durch den ESM sah der Gerichtshof keine von Art. 125 AEUV verbotene Haftung oder Eintreten für Verbindlichkeiten des ESM-Mitgliedstaats. Im Sinne der Zwecksetzung des Art. 125 AEUV, die Haushaltsdisziplin der Mitgliedstaaten zu wahren, stützte der Gerichtshof seine Erwägungen insbesondere darauf, dass sowohl der neu eingefügte Art. 136 Abs. 3 AEUV als auch die Bestimmungen des ESM-Vertrages selbst eine Aktivierung einer Finanzhilfe nur dann vorsehen, wenn sie für die Wahrung der Finanzstabilität des gesamten Euro-Währungsgebiets unabdingbar ist und strengen Auflagen unterliegt. ${ }^{35}$

Das Bundesverfassungsgericht hatte bereits in seiner Entscheidung zum einstweiligen Rechtsschutz vom 12. September 2012 den Erlass einstweiliger Anordnungen bzgl. der Gesetze zur Ratifizierung des ESM, des Fiskalvertrages sowie zum Beschluss des Europäischen Rates zur Änderung des Art. 136 AEUV abgelehnt. Für den ESM-Vertrag hatte das BVerfG allerdings eine Ratifizierung nur mit der Maßgabe für zulässig erklärt, dass völkerrechtlich sichergestellt wird, dass die in Art. 8 Abs. 5 Satz 1 ESM-Vertrag enthaltene Haftungsbeschränkung auf den Anteil Deutschlands am genehmigten Stammkapital in keinem Fall überschritten werden dürfe und eine umfassende Unterrichtung des Bundestages und Bundesrates gewährleistet werde. Zur Umsetzung dieser Maßgabe erfolgte auf Initiative Deutschlands eine völkerrechtlich verbindliche, gemeinsame interpretative Erklärung der ESM-Mitgliedstaaten, in der die vom BVerfG geforderten interpretativen Klarstellungen der betroffenen ESM-Artikel niedergelegt wurden. Im Anschluss wurden der ESM-Vertrag und Fiskal-Vertrag ratifiziert. In seinem Urteil vom 18. März 2014 hat das Bundesverfassungsgericht diese völkerrechtliche Lösung im Grundsatz gebilligt und im Ergebnis die Beteiligung Deutschlands am ESM für verfassungsgemäß befunden. ${ }^{36}$

Das beschriebene Vorgehen der Mitgliedstaaten ist im Ergebnis nicht als Missachtung bzw. „Verweigerung“ des Rechts einzustufen. Denn die Mitgliedstaaten standen mit der sich im Herbst 2009 und 2010 abzeichnenden Staatsschuldenkrise 
Griechenlands und den zu befürchtenden Auswirkungen auch auf weitere Staaten der Euro-Zone vor einer Situation, in der sich die Frage der Zulässigkeit finanzieller Unterstützung erstmals so zugespitzt stellte. Damit einher ging die Notwendigkeit, den Inhalt des Bail-out-Verbots gem. Art. 125 AEUV - ohne damals einschlägige Rechtsprechung - dahingehend auszuloten, ob dieses Verbot auch die Gewährung bilateraler Kredite unter den gegebenen Umständen erfassen sollte. Diese Frage haben die Mitgliedstaaten geprüft und im Ergebnis verneint. Um jedoch explizit klar zu stellen, dass solche finanziellen Hilfen nur als ultima ratio und nur bei Sicherstellung der Haushaltsdisziplin des betroffenen Mitgliedstaats zulässig sein sollten, haben die Mitgliedstaaten sowohl bei Ausgestaltung des ESM als auch durch Einfügung des Art. 136 Abs. 3 AEUV die Gewährung der Unterstützung an strenge Voraussetzungen gebunden. Dies zeigt, dass die Mitgliedstaaten und die EU gerade bemüht waren, sich rechtskonform zu verhalten und dies durch zusätzliche Maßnahmen auch sicherzustellen.

Selbstverständlich lässt sich dogmatisch weiter darüber streiten, ob die Maßnahmen im Einklang mit Art. 125 AEUV stehen. In der europarechtlichen Literatur finden sich hierzu einige kritische Stimmen. ${ }^{37}$ Wenn es jedoch darum geht, ob die Mitgliedstaaten noch im Sinne einer Rechtsgemeinschaft agieren ${ }^{38}$, ist maßgeblich, dass die betreffenden Maßnahmen letztlich sowohl vom Europäischen Gerichtshof als auch vom Bundesverfassungsgericht gebilligt worden sind.

\section{Migration}

Auch im Bereich der Migration, insbesondere im Rahmen der Flüchtlingskrise seit Sommer 2015 stellte sich die Frage, ob Mitgliedstaaten sich in unzulässiger Weise der Befolgung geltenden Rechts verweigern.

Vor allem die Regelungen der Dublin III-Verordnung wurden seit Beginn der Flüchtlingsströme 2015 auf eine harte Bewährungsprobe gestellt. Das der Verordnung zugrunde liegende Prinzip, dass im Regelfall derjenige Mitgliedstaat für die Bearbeitung eines Asylantrags zuständig ist, in den der Flüchtling erstmals von einem Drittstaat in die EU einreist, wurde in der Krisenzeit von den Mitgliedstaaten an den EU-Außengrenzen zu großen Teilen nicht mehr umgesetzt. Vielmehr wurden Migranten aus Italien und weiteren Staaten weitergeleitet an Mitgliedstaaten, die das Ziel der Flüchtlinge waren - insbesondere Österreich und Deutschland.

Mit dieser Situation etwa war die Große Kammer des Gerichtshofs in den Urteilen vom 26. Juli 2017 in den Rechtssachen A.S. (C-490/16) und Jafari (C-646/16) im Hinblick auf Weiterleitungen aus Kroatien nach Slowenien bzw. Österreich be-

37 Vgl. hierzu etwa M. Potacs, Die Europäische Wirtschafts- und Währungsunion und das Solidaritätsprinzip, EuR 2013, S. 133, $136 \mathrm{ff}$.

38 Dies bejahend auch F. Mayer, Die Europäische Union als Rechtsgemeinschaft - ein überholtes Narrativ?, in: Franzius/Mayer/Neyer (Hrsg.), Die Neuerfindung Europas - Bedeutung und Gehalte von Narrativen für die europäische Integration, 2019, S. 111, $116 \mathrm{f}$. 
fasst. Die Zuständigkeit für die Prüfung der Gewährung internationalen Schutzes nach der Dublin III-Verordnung hing in diesen Fällen insbesondere von der Auslegung der Artikel 12 und 13 der Verordnung ab, die demjenigen Mitgliedstaat die Zuständigkeit zuschreiben, der bei der erstmaligen Einreise aus einem Drittstaat entweder ein Visum erteilt hat, oder in den der Drittstaatsangehörige ,illegal“ eingereist ist. Der Gerichtshof entschied in der Rechtssache Jafari, dass nicht etwa das Vorliegen eines „Visums“ im Sinne von Art. 12 fingiert werden könne, wenn ein solches nicht förmlich erteilt wurde, sondern die Behörden eines Mitgliedstaats , in einer Situation, in der sie mit der Ankunft einer außergewöhnlich hohen Zahl von Drittstaatsangehörigen konfrontiert sind, die durch diesen Mitgliedstaat durchreisen möchten, um in einem anderen Mitgliedstaat internationalen Schutz zu beantragen, die Einreise der Drittstaatsangehörigen dulden". 39 Jedoch sei in einer solchen Konstellation davon auszugehen, dass der einreisende Drittstaatsangehörige ,die Grenze des erstgenannten Mitgliedstaats im Sinne von Art. 13 Abs. 1 , illegal überschritten ' hat ${ }^{\text {'40. }}$. Danach war Kroatien für die Prüfung der Gewährung subsidiären Schutzes grundsätzlich zuständig, wobei der Gerichtshof auch darauf hinwies, dass nach Art. 3 Abs. 2 Unterabs. 2 der Dublin-III-Verordnung und Art. 4 der Charta der Grundrechte der Europäischen Union eine Person, die internationalen Schutz beantragt hat, ,nicht an den zuständigen Mitgliedstaat überstellt werden darf, wenn die Überstellung für sie mit der tatsächlichen Gefahr verbunden ist, eine unmenschliche oder erniedrigende Behandlung im Sinne von Art. 4 der Charta zu erleiden" ${ }^{\prime 4}$. Eine Überstellung könne daher nicht vorgenommen werden, ,wenn im zuständigen Mitgliedstaat infolge der Ankunft einer außergewöhnlich hohen Zahl internationalen Schutz begehrender Drittstaatsangehöriger eine solche Gefahr besteht ${ }^{642}$. Im Ergebnis hat der Gerichtshof damit festgestellt, dass die bloße Durchleitung der Flüchtlinge nach Slowenien bzw. Österreich einen Verstoß Kroatiens gegen die entsprechenden Regelungen der Dublin III-Verordnung darstellte. Aus dem Hinweis des Gerichtshofs auf den außergewöhnlichen Ansturm von Flüchtlingen lässt sich jedoch möglicherweise auch wenn der Gerichtshof dies nicht unmittelbar so formuliert - eine gewisse krisenbedingte „Entschuldigung“ für das Verhalten Kroatiens herleiten.

Die vielfach kritisierte Aufnahme einer hohen Zahl von Flüchtlingen durch Deutschland im Jahr 2015 dürfte zumindest mittelbar durch den Gerichtshof in seinem Urteil in der Rs. 646/17 (Jafari) als europarechtskonform gebilligt worden sein. Deutsche Maßnahmen sind zwar nicht Gegenstand dieser Entscheidung. Jedoch verweist der Gerichtshof im Zusammenhang mit der Bestätigung, dass eine „illegale“ Einreise nach Kroatien und damit eine Zuständigkeit Kroatiens für die

39 EuGH, Urt. v. 26.7.2017, Rs. C-646/16 (Khadija Jafari u.a.), ECLI:EU:C:2017:586, Rn. 58.

40 EuGH, Urt. v. 26.7.2017, Rs. C-646/16 (Khadija Jafari u.a.), ECLI:EU:C:2017:586, Rn. 102; EuGH, Urt. v. 26.7.2017, Rs. C-490/16 (A.S.), ECLI:EU:C:2017:585, Rn. 39.

41 EuGH, Urt. v. 16.2.2017, C-578/16 PPU (C. K. u.a.), ECLI:EU:C:2017:127, Rn. 65.

42 EuGH, Urt. v. 26.7.2017, Rs. C-646/16 (Khadija Jafari u.a.), ECLI:EU:C:2017:586, Rn. 101; EuGH, Urt. v. 26.7.2017, Rs. C-490/16 (A.S.), ECLI:EU:C:2017:585, Rn. 41. 
Prüfung der Anträge auf internationalen Schutz bestand, darauf, dass die Aufnahme einer außergewöhnlich hohen Zahl von Schutzsuchenden durch diesen Staat auch dadurch erleichtert werden kann, „dass andere Mitgliedstaaten einseitig oder in Abstimmung mit dem betreffenden Mitgliedstaat, im Geist der Solidarität, der im Einklang mit Art. 80 AEUV der Dublin III-Verordnung zugrunde liegt", von dem Selbsteintrittsrecht nach Art. 17 Abs. 1 der Dublin III-VO Gebrauch machen können. ${ }^{43}$ Noch nicht Gegenstand eines Verfahrens vor dem Gerichtshof sind zwischenzeitlich quasi dauerhaft wiedereingeführte Grenzkontrollen durch einige Mitgliedstaaten und die Frage, ob diese mit dem Schengener Grenzkodex vereinbar sind. ${ }^{44}$ Dies gilt entsprechend für die Frage, ob eine Zurückweisung an einer EU-Binnengrenze durch einen Mitgliedstaat mit den Regelungen der Dublin III.VO vereinbar ist. ${ }^{45}$

Weitere mitgliedstaatliche Widerstände gegen die Befolgung von Rechtsakten betreffen die Frage der Verteilung von Flüchtlingen innerhalb der EU. Im September 2015 erließ der Rat der EU gestützt auf Art. 78 Abs. 3 AEUV zur Bewältigung des hohen Zustroms von Schutzsuchenden einen Beschluss über vorläufige Maßnahmen im Bereich des internationalen Schutzes zugunsten von Italien und Griechenland. ${ }^{46}$ Er sah vor, dass 120.000 schutzbedürftige Personen über einen Zeitraum von 2 Jahren aus diesen beiden Mitgliedstaaten in die anderen Mitgliedstaaten der Union verteilt werden sollten. Ungarn und die Slowakei, unterstützt durch Polen, beantragten beim Gerichtshof, den Beschluss für nichtig zu erklären. Während die Slowakei einige wenige Flüchtlinge aus dem Beschluss aufgenommen hat ${ }^{47}$, weigerten sich Ungarn, Polen und Tschechien insgesamt, dem angefochtenen Beschluss nachzukommen. Die Kommission hat vor diesem Hintergrund im Juni 2017 Vertragsverletzungsverfahren gegen diese drei Mitgliedstaaten eingelei-

43 EuGH, Urt. v. 26.7.2017, Rs. C-646/16 (Khadija Jafari u.a.), ECLI:EU:C:2017:586, Rn. 100. Vgl. aber kritisch zur Ausübung des Selbsteintrittsrechts durch Deutschland z.B. M. Wendel, Asylrechtlicher Selbsteintritt und Flüchtlingskrise - Zugleich ein Beitrag zu den Grenzen administrativer Entscheidungsspielräume im Mehrebenensystem, JZ 2016, S. 332, $337 \mathrm{ff}$.

44 Dies gilt etwa für Deutschland, Österreich und Dänemark. Siehe z.B. tagesschau.de vom 12.4.2019, Deutschland verlängert Grenzkontrollen, https://www.tagesschau.de/inland/deutschland-grenzkontrollen-113.html; https://www.dw.com/de/\%C3\%B6sterreich-verl\%C3\%A4ngert-grenzkontrollen-bis-mai-2019/a-45848406. Siehe auch dw.com vom 12.10.2018, Weiter Grenzkontrollen in der EU, https:/www.dw.com/de/weiter-grenz kontrollen-in-der-eu/a-45863607.

45 Siehe kritisch hierzu z.B. C. Hruschka, Dublin ist kein 5-Minuten-Verfahren, Verfassungsblog vom 23.6.2018, https://verfassungsblog.de/dublin-ist-kein-5-minuten-verfahren-zu-zurueckweisungen-an-der-gren ze/. A.A. (die rechtliche Zulässigkeit der Zurückweisung bejahend) z.B. M. Möstl, Verfassungsfragen der Flüchtlingskrise 2015/16, AöR 2017, S. 175, 221 f.; A. Peukert/C. Hillgruber/U. Foerste/H. Putzke, Einreisen lassen oder zurückweisen? Was gebietet das Recht in der Flüchtlingskrise an der deutschen Staatsgrenze?, ZAR 2016, S. 131, $132 \mathrm{f}$.

46 Beschluss (EU) 2015/1601 des Rates vom 22.9.2015 zur Einführung von vorläufige Maßnahmen im Bereich des internationalen Schutzes zugunsten von Italien und Griechenland, ABl. EU 2015 L 248/80.

47 Vgl. Europäische Kommission, 14th Report on Relocation and Resettlement vom 26.7.2017, COM (2017) 405 final, Annex 3: 16 Flüchtlinge aus Griechenland; siehe Spiegel-online vom 1.8.2017: Slowakei nimmt 60 Flüchtlinge auf (50 aus Italien, weitere 10 aus Griechenland), http://www.spiegel.de/politik/ausland/slowakeinimmt-60-fluechtlinge-auf-a-1160930.html. 
tet $^{48}$ und im Dezember 2017 Klage ${ }^{49}$ erhoben. Die Klagen Ungarns und der Slowakei gegen den Ratsbeschluss zur Flüchtlingsverteilung vom 22.9.2015 hat der Gerichtshof am 6. September 2017 in vollem Umfang abgewiesen. 50

Der Gerichtshof bekräftigte in seinem Urteil, dass der Beschluss mit qualifizierter Mehrheit in einem ordnungsgemäßen Verfahren nach Art. 78 Abs. 3 AEUV gefasst wurde und auch den Anforderungen des europarechtlichen Verhältnismäßigkeitsprinzips standhält; in Bezug auf die Verhältnismäßigkeit des beschlossenen Verteilungsmechanismus wies der Gerichtshof auf den weiten Beurteilungsspielraum des Unionsgesetzgebers hin. ${ }^{51}$

Die ersten Reaktionen auf dieses Urteil sind in der Slowakei und Ungarn unterschiedlich ausgefallen. Die Slowakei hat nach Verkündung des Urteils signalisiert, dass sie es grundsätzlich akzeptiere. ${ }^{52}$ Ungarn dagegen wies in einer ersten Stellungnahme des Außenministers Peter Szijjarto die Entscheidung scharf als ein politisches, ,inakzeptables“ Urteil, das die Sicherheit und die Zukunft ganz Europas gefährde, zurück. Den EU-Beschluss erachte er weiterhin als ,nicht verpflichtend". ${ }^{53}$ Auch Polen und Tschechien verkündeten, dass sie weiterhin keine Flüchtlinge aus dem Verteilungsbeschluss aufnehmen würden.

Insbesondere die von Ungarn unmittelbar nach dem Urteil des Gerichtshofs eingenommene Haltung erscheint bedenklich, da sie den Willen signalisiert, die Entscheidung des Gerichtshofs zu ignorieren. Viktor Orbán wurde in Pressemeldungen dahingehend zitiert, dass Ungarn als Mitgliedsland der EU zwar ,die Verträge und Entscheidungen des Gerichts anerkennen" müsse, das Urteil aber keinen Grund dafür darstelle, ,die Politik der Ablehnung von Migranten zu ändern“.54 Die Frage, die sich nun stellt, betrifft das weitere Vorgehen der EU. Insoweit sind durchaus noch nicht alle Möglichkeiten ausgereizt. Die Kommission hat, wie oben ausgeführt, bereits Vertragsverletzungsverfahren gegen Ungarn, Polen und

48 Vgl. Pressemitteilung der Europäischen Kommission, IP/17/1607 vom 14. 06.2017; siehe auch Zeit online vom 13. Juni 2017, www.zeit.de/politik/ausland/2017-06/fluechtlinge-eu-geht-rechtlich-gegen-ungarn-polenund-tschechien-vor.

49 Siehe Meldung der Europäischen Kommission, abrufbar unter https://ec.europa.eu/germany/news/20171207vertragsverletzungen-kommission-verklagt-tschechische-republik-ungarn-und-polen_de.

$50 \mathrm{EuGH}$, Urteil vom 6.9.2017, verb. Rs. C-643/15 und 647/15 (Slowakei und Ungarn/Rat), ECLI:EU:C:2017:631; GA Bot, Schlussanträge vom 26.7.2017 in den verb. Rs. C-643/15 und C-647/15 (Slowakische Republik und Ungarn/Rat der Europäischen Union), ECLI:EU:C:2017:618.

51 EuGH, Urteil vom 6.9.2017, verb. Rs. C-643/15 und 647/15 (Slowakei und Ungarn/Rat). ECLI:EU:C:2017:631, Rn. 207.

52 FAZ vom 7.9.2017, S. Löwenstein, Orbán schäumt, Fico moderiert - Reaktionen auf das EuGH-Urteil.

53 FAZ vom 7.9.2017, Ungarn und Slowakei müssen Flüchtlinge aufnehmen - EuGH weist Klage gegen Quoten ab/Budapest: Empörend/Pressburg: Voll respektieren.

54 Siehe etwa BR24 v. 8.9.2017, https://www.br.de/nachrichten/deutschland-welt/ungarn-will-weiter-keine-fluec htlinge-aufnehmen,QUd8Rh5; siehe hierzu auch FAZ v. 12.9.2017, Nach EuGH-Urteil : Merkel will Orbans Flüchtlingspolitik nicht akzeptieren, „Ungarns Ministerpräsident will trotz EuGH-Entscheidung nicht mehr Flüchtlinge aufnehmen. Bundeskanzlerin Merkel sieht darin Europa als ,, Raum des Rechts “ in Frage gestellt [...]“, https://www.faz.net/aktuell/politik/nach-eugh-urteil-merkel-will-orbans-fluechtlingspolitik-nicht-akzep tieren-15194348.html. 
Tschechien wegen der Nichtumsetzung des Verteilungsbeschlusses eingeleitet. ${ }^{55}$ In diesen Vertragsverletzungsverfahren fand am 15. Mai 2019 eine mündliche Verhandlung statt. Wenn ein Urteil des Gerichtshofs in diesen Vertragsverletzungsverfahren vorliegt, kann die Kommission, soweit die beklagten Mitgliedstaaten nicht selbst einlenken sollten, ein Zwangsgeldverfahren einleiten, an dessen Ende die Verhängung finanzieller Sanktionen stehen könnte. Dabei ist allerdings zu berücksichtigen, dass der Beschluss über die Umverteilung der Asylbewerber bis zum 26. September 2017 befristet war. Es ist daher fraglich, ob mit Ablauf dieser Frist eine Verpflichtung zur Aufnahme von Flüchtlingen aus diesem Beschluss noch mittels eines Zwangsgelds erzwungen werden kann. ${ }^{56}$

Wenn Ungarn sich weiter seinen Verpflichtungen zur Aufnahme von Flüchtlingen widersetzt und seine zurückweisende Haltung auch im Falle eines Urteils des Gerichtshofs im Vertragsverletzungs- oder gar Zwangsgeldverfahren beibehält, würde dies in der Tat die Rechtsgemeinschaft bedrohen. Eine Lösung dieser prekären Situation muss allerdings über die in jedem Fall notwendige Durchsetzung des bestehenden Rechts hinausgehen. Denn ebenso wie dies entsprechend bei Beginn der Finanzkrise der Fall war, kranken die Regelungen zur Migration aus Drittstaaten, insbesondere die Dublin III-Verordnung, daran, dass sie selbst keine angemessenen Lösungen zur Bewältigung der Flüchtlingsströme, die in 2015 einsetzten, bereit halten. ${ }^{57}$ Die Mitgliedstaaten und die Union hatten daher in einer politisch äußerst schwierigen Situation kurzfristig zu entscheiden und in erster Linie vorübergehende Notmaßnahmen zu treffen. Eine grundsätzliche Reform des Systems - die auch die designierte Kommissionspräsidentin Ursula von der Leyen als eine der Prioritäten der Kommission im Zeitraum 2019 bis 2024 nennt $^{58}$ - ist vor diesem Hintergrund dringend erforderlich.

\section{Durchsetzung des Rechtsstaatsprinzips}

Ein gravierender Vorwurf mitgliedstaatlichen Fehlverhaltens knüpft an die Verletzung des Rechtsstaatsprinzips an. Insbesondere gegenüber Polen und Ungarn,

55 Die Kommission hat am 19.7.2018 zudem beschlossen, gegen Ungarn Klage vor dem EuGH zu erheben, weil die Asyl- und Rückführungsvorschriften Ungarns nicht mit EU-Recht vereinbar sind (siehe Pressemitteilung der KOM, https://ec.europa.eu/germany/news/20180719-kommission-verklagt-ungarn-wegen-asylpolitik_de). Siehe auch das am selben Tag gegen Ungarn eingeleitete Vertragsverletzungsverfahren wegen eines Gesetzgebungspakets, das Hilfeleistungen bei Asyl- und Aufenthaltsanträgen unter Strafe stellt; hierzu hat die Kommission am 24.1.2019 eine mit Gründen versehene Stellungnahme beschlossen (siehe Pressemitteilung der KOM, http://europa.eu/rapid/press-release MEMO-19-462 de.htm). In diesem Verfahren hat die KOM am 24.7.2019 Klage beim EuGH erhoben, https://europa.eu/rapid/press-release_IP-19-4260_de.htm.

56 Siehe hierzu auch C. Hruschka, EuGH verhandelt über Flüchtlingsverteilung in Europa: Kommen Polen, Tschechien und Ungarn damit durch?, in: Legal Tribune Online, 14.5.2019 (https://www.lto/persistent/a_id/35 377).

57 Siehe in diesem Sinne auch z.B. A. Weber, Europäisches Rechtsdenken in der Krise?, DÖV 2017, S. 741, 745 f. mwN; Siehe hierzu auch D. Thym, Migrationsverwaltungsrecht 2010, S. 365.

58 Siehe von der Leyen, A Union that strives for more - My agenda for Europe, Political Guidelines for the next European Commission 2019-2024, https://ec.europa.eu/commission/sites/beta-political/files/political-guidelin es-next-commission_en.pdf. 
aber auch weiteren Mitgliedstaaten, wird der Vorwurf erhoben, dass sie die in Art. 2 EUV niedergelegten Werte der EU, insbesondere das dort genannte Rechtsstaatsprinzip, missachten würden. Die Besonderheit liegt hier darin, dass es sich zum einen bei den kritisierten Regelungen - etwa zur Besetzung der nationalen Gerichte - um auf den ersten Blick innerstaatliche Vorschriften handelt, bei denen der Bezug zum EU-Recht jedenfalls nicht offensichtlich ist. Zum anderen besteht für die Ahndung von Verstößen des Art. 2 EUV ein spezifisches Verfahren nach Art. 7 EUV, so dass sich die Frage stellt, ob und inwieweit Vertragsverletzungsverfahren (ergänzend) herangezogen werden können.

\section{Verfahren nach Art. 7 EUV und Rechtsstaatsdialog}

Zur Sanktionierung mangelnder Rechtsstaatlichkeit, die als grundlegender Wert der Europäischer Union Eingang in Art. 2 EUV gefunden hat, wurde in Art. 7 EUV ein spezifisches Verfahren eingeführt. Danach kann der Rat - nach Durchführung des in Art. 7 Abs. 1 EUV geregelten Verfahrens - feststellen, dass ,die eindeutige Gefahr einer schwerwiegenden Verletzung" der Rechtsstaatlichkeit durch einen Mitgliedstaat besteht. In einer zweiten Stufe besteht die Möglichkeit, dass der Europäische Rat eine „schwerwiegende und anhaltende Verletzung“ eines der in Art. 2 EUV genannten Werte konstatiert. Diese Feststellung, die mögliche Sanktionen vorbereitet, setzt nach Art. 7 Abs. 2 EUV Einstimmigkeit im Europäischen Rat voraus. Wurde sie getroffen, kann der Rat mit qualifizierter Mehrheit gemäß Art. 7 Abs. 3 EUV bestimmte Sanktionen, wie etwa die Aussetzung der Stimmrechte des Vertreters der Regierung des betroffenen Mitgliedstaats, beschließen.

Die Kommission hat am 20. Dezember 2017 im Hinblick auf die polnische Gesetzgebung zur Änderung der dortigen Struktur des Justizsystems ein Verfahren nach Art. 7 Abs. 1 EUV gegen Polen eröffnet. ${ }^{59}$ Nach Anhörungen Polens im Rat wurde dieses Verfahren zunächst nicht weiterbetrieben, auch vor dem Hintergrund der parallel beim Europäischen Gerichtshof anhängigen Vertragsverletzungsverfahren zur Absenkung des Pensionsalters polnischer Richter am Obersten Gericht. Im Falle Ungarns wurde das Verfahren nach Art. 7 Abs. 1 EUV erstmals durch das Europäische Parlament eingeleitet. Mit Entschließung des Europäischen Parlaments vom 12. September 2018, die mit 448 Stimmen bei 197 Gegenstimmen und 48 Enthaltungen angenommen wurde, forderte das Europäische Parlament den Rat auf, ,festzustellen, ob die eindeutige Gefahr einer schwerwiegenden Verletzung der in Artikel 2 EUV genannten Werte durch Ungarn besteht, und diesbezüglich geeignete Empfehlungen an Ungarn zu richten".60 Die ungarische Regie-

59 Europäische Kommission, Begründeter Vorschlag gem. Art. 7 Abs. 1 EUV vom 20.12.2017, COM (2017) 835 final. Siehe hierzu auch z.B. U. Karpenstein, Editorial - Europa zeigt Zähne, Dreimal Polen, dreimal Neuland: Kommission und EuGH machen nun ernst, EuZW 2018, S. $97 \mathrm{ff}$.

60 Entschließung des Europäischen Parlaments vom 12. September 2018 zu einem Vorschlag, mit dem der Rat aufgefordert wird, im Einklang mit Artikel 7 Absatz 1 des Vertrags über die Europäische Union festzustellen, 
rung hat am 17. Oktober 2018 Klage gegen das Europäische Parlament erhoben, mit dem Ziel, die Entschließung vom 12. September für nichtig erklären zu lassen. Ihre Klage stützt die ungarische Regierung vor allem auf mutmaßliche Verfahrensfehler bei der Abstimmung über die Entschließung sowie auf den Vorwurf der Verletzung des Prinzips der loyalen Zusammenarbeit der Union und ihrer Mitgliedstaaten einerseits sowie der Unionsorgane untereinander andererseits, des Vertrauensschutzes sowie der Rechtssicherheit, da die Entschließung Feststellungen unter Berufung auf noch anhängige bzw. abgeschlossene Vertragsverletzungsverfahren treffe. ${ }^{61}$

Dem besonderen Verfahren des Art. 7 EUV, das aufgrund des erwähnten Einstimmigkeitsprinzips in seinem Absatz 2 in der Praxis politisch nur schwer durchsetzbar ist, hatte die Europäische Kommission im März 2014 den sog. Rechtsstaatsdialog vorangestellt ${ }^{62}$, den sie gegenüber Polen im Hinblick auf die dortige Justizreform im Januar 2016 eingeleitet hatte.

Der Rechtsstaatsdialog soll - ergänzend zum Verfahren nach Art. 7 EUV und etwaigen Vertragsverletzungsverfahren - ein Instrument zur Behandlung systematischer Verletzungen des in Art. 2 EUV niedergelegten Rechtsstaatsprinzips in den EU-Mitgliedstaaten bieten. Er ermöglicht der Kommission - auch wenn die Schwelle eines Art. 7 EUV-Verfahrens noch nicht erreicht ist - frühzeitig in den Dialog mit einem Mitgliedstaat zu treten, der nach ihrer Auffassung Prinzipien der Rechtsstaatlichkeit systemisch verletzt. Die Frage, ob die EU eine Kompetenz für einen sog. Rechtsdialog in Bezug auf einen solchen, doch möglicherweise primär innerstaatlichen Vorgang besitzt, ist bis heute nicht abschließend geklärt. ${ }^{63}$ Möglicherweise lässt sich argumentieren, dass der Rechtsstaatsdialog an sich keiner Rechtsgrundlage bedarf, da es in erster Linie um einen politischen Dialog, nicht aber um den Erlass rechtlich bindender Rechtsakte geht. Im Ergebnis handelt es sich jedoch um einen schwierigen rechtlichen Balanceakt zwischen dem Ziel einer Bewahrung und Stärkung gemeinsamer Werte einerseits und der Achtung staatlicher Souveränität andererseits. ${ }^{64}$

dass die eindeutige Gefahr einer schwerwiegenden Verletzung der Werte, auf die sich die Union gründet, durch Ungarn besteht (2017/2131(INL), P8_TA-PROV (2018) 0340).

61 Klage Ungarn gegen Europäisches Parlament, eingereicht am 17.10.2018, Rs. C-650/18; http://curia.europa.e $\mathrm{u} /$ juris/document/document.jsf?text $=\&$ docid $=208326 \&$ pageIndex $=0 \&$ doclang $=$ DE $\&$ mode $=$ req $\&$ dir $=\&$ occ $=$ fir st\&part=1; BeckEuRS 2018, 577406.

62 Europäische Kommission, Mitteilung vom 11.3.2014, Ein neuer EU-Rahmen zur Stärkung des Rechtsstaatsprinzips, 11.3.2014 (COM (2014) 158 final). Siehe auch Pressemitteilung der Europäischen Kommission vom 11.3.2014 (IP/14/237).

63 Siehe hierzu etwa V. Skouris, Die Rechtsstaatlichkeit in der Europäischen Union, EuR 2015 Beiheft 2, S. 9 , 17; siehe auch F. Schorkopf, Wertesicherung in der Europäischen Union. Prävention, Quarantäne und Aufsicht als Bausteine eines Rechts der Verfassungskrise?, EuR 2016, S. 147, 160 (mit Verweis u.a. auch auf Stellungnahme des Juristischen Dienstes des Rates, der eine Rechtsgrundlage in den EU-Verträgen verneint hatte, Opinion of the Legal Service, Dok. Nr. 10296/14, Ziffer 15-27). Vgl. auch A. Weber, Europäisches Rechtsdenken in der Krise?, DÖV 2017, S. 741, 747.

64 Dies gilt auch im Hinblick auf weitere von der Kommission in Aussicht gestellte Maßnahmen, die sie in ihrer Mitteilung vom 3. April 2019 (Europäische Kommission, Mitteilung vom 3.04.2019, Die weitere Stärkung der Rechtsstaatlichkeit in der Union - Aktuelle Lage und mögliche nächste Schritte, KOM (2019) 163 final) nennt. Bezüglich der dort vorgeschlagenen Förderung der Rechtsstaatlichkeit im Sinne eines stärkeren Auf- 


\section{Vertragsverletzungsverfahren zur Durchsetzung der Rechtsstaatlichkeit}

Die Frage, ob mit mitgliedstaatlichem Verhalten, das eine Gefährdung der Rechtsstaatlichkeit in diesem Staat bewirkt, (auch) eine Verletzung von EU-Recht einhergeht, stellt sich zugespitzt bei der Entscheidung, ob bei einem Verstoß gegen rechtsstaatliche Grundsätze ein Vertragsverletzungsverfahren gem. Art. 258 AEUV gegen den betreffenden Mitgliedstaat eingeleitet werden kann.

Gegen Ungarn und Polen hat die Kommission jeweils Vertragsverletzungsverfahren in Bezug auf gesetzliche Regelungen zur Absenkung des Ruhestandsalters u.a. für Richter eingeleitet. Beide Verfahren wurden auf die Verletzung von Unionsrecht gestützt. Im Falle Ungarns hat der Europäische Gerichtshof einen Verstoß gegen Art. 2 und Art. 6 Abs. 1 der Richtlinie 2000/78/EG im Ergebnis bejaht, da die unvermittelte Herabsetzung des Pensionsalters für Richter, Staatsanwälte und Notare von 70 Jahre auf 62 Jahre - ohne Übergangsfristen vorzusehen - eine unverhältnismäßige Ungleichbehandlung aufgrund des Alters bewirke. ${ }^{65}$ Ungarn ist diesem Urteil mit einer Gesetzesänderung nachgekommen, wonach das Pensionsalter für Richter nun auf 65 Jahre nach einer zehnjährigen Übergangszeit festgesetzt und Vorkehrungen für die Wiedereinsetzung von Richtern vorgesehen wurden, die unrechtmäßig entlassen wurden. Vizepräsidentin der Kommission $V i$ vian Reding erkannte diese Gesetzesänderung an, verwies allerdings darauf, dass eine vollständige Umsetzung des Urteils vom 6. November 2012 nicht nur eine gesetzliche Maßnahme, sondern auch die tatsächliche Wiedereinsetzung unrechtmäßig entlassener Richterinnen und Richter verlange. ${ }^{66}$ Im Falle Ungarns erfolgte dies allerdings nicht in allen Fällen, zumindest nicht im Sinne einer Wiedereinsetzung auf die ursprünglichen Positionen. ${ }^{67}$

Im Vertragsverletzungsverfahren gegen Polen, das die polnischen Bestimmungen zur Senkung des Ruhestandsalters der Richter am Obersten Gericht vom 3. April

baus von Wissen und Schaffung einer gemeinsamen Kultur der Rechtsstaatlichkeit in den EU-Mitgliedstaaten dürfte eine eigene Rechtsgrundlage noch nicht erforderlich sein. Schon aber die Überlegungen der Kommission zu einer besseren Vorbeugung gegen Rechtsstaatlichkeitsprobleme in den Mitgliedstaaten und einer stärkeren Zusammenarbeit bzw. Unterstützung durch die EU etwa auch mit Hilfe von Datenerhebungen in allen Mitgliedstaaten zu Bereichen der „Unabhängigkeit der Justiz, die Qualität der Verwaltung, die Korruptionsbekämpfungsstrategien, die Transparenz des Legislativprozesses und die Verbesserung der Rechtsetzung“" (Europäische Kommission, a.a.O. S. 14) als Grundlage für einen besseren Dialog zwischen EU und Mitgliedstaaten lassen zumindest Zweifel an kompetenzrechtlichen Zulässigkeit aufkommen.

65 EuGH, Urt. v. 6.11.2012, Rs. C-286/12 (Kommission/Ungarn), ECLI:EU:C:2012:687, Rn. 68 ff. Siehe auch EuGH, Urt. v. 8.4.2014, Rs. C-288/12 (Kommission/Ungarn), ECLI:EU:C:2014:237 (Unabhängigkeit des ungarischen Datenschutzbeauftragten), Verletzung der Datenschutzrichtlinie 95/46/EG. Nicht die Rechtsstaatlichkeit im engeren Sinne betreffende, aber damit im Zusammenhang stehende Vertragsverletzungsverfahren gegen Ungarn betreffen etwa das Gesetz über aus dem Ausland finanzierter Nichtregierungsorganisationen (Klage der Kommission vom 6.2.2018, Rs. C-78/18) und Änderungen am Hochschulgesetz (Klage vom 1.2.2018, Rs. C-66/18).

66 Pressemitteilung der Kommission vom 17.3.2013, SPEECH/13/324 (http://europa.eu/rapid/pressrelease_SPE ECH-13-324_en.htm); siehe zu Ungarn aus europarechtlicher Sicht auch die Veröffentlichung des Bundestags, Gesetzes- und Verfassungsänderungen in Ungarn seit 2010 aus Sicht des Rechts der Europäischen Union, PE 6 - 3000 - 46/13 (www.bundestag.de/resource/blob/408304/c4c6c0068c9c5299545eaa7ee4ecb0ea/pe6-046-13-pdf-data.pdf).

67 Siehe A. Eppler, Die „Rechtsstaatskrise“ der EU: Verderben zu viele Köche den Brei?, ZfP 2016, S. 406, 420. 
2018 angreift, hatte der EuGH Polen in einer einstweiligen Anordnung durch Beschluss der Vizepräsidentin Rosario Silva de Lapuerta vom 19. Oktober 2018 angewiesen, die Anwendung der umstrittenen Regelungen unverzüglich auszusetzen. ${ }^{68}$ Diese Eilentscheidung erging aufgrund der unmittelbaren Gefahr eines schweren und nicht wiedergutzumachenden Schadens im Hinblick auf den Grundsatz des wirksamen gerichtlichen Rechtsschutzes noch bevor Polen seine Stellungnahme eingereicht hatte. Bereits nach dieser Entscheidung rief die Präsidentin des Obersten Gerichtshofs Polens, Malgorzata Gersdorf, die zwangspensionierten Richter zurück in den Dienst. ${ }^{69}$ Einige der Richter erschienen am 22. Oktober daraufhin im Dienst. ${ }^{70}$ Nach Anhörung Polens bestätigte schließlich der Gerichtshof durch Beschluss der Großen Kammer vom 17. Dezember 2018 die im Oktober getroffene einstweilige Anordnung. ${ }^{71}$ Polens Regierung hat daraufhin die umstrittene Zwangspensionierung aufgehoben.

Im Hauptsacheverfahren in dieser Rechtssache hat Generalanwalt Tanchev am 11. April 2019 seine Schlussanträge vorgelegt. ${ }^{72}$ Er kommt darin zu dem Schluss, dass die Rügen der Kommission, soweit sie auf Art. 47 der Grundrechte-Charta gegründet sind, unzulässig seien, da die Kommission nicht dargelegt habe, dass die fraglichen Maßnahmen Polens der Durchführung von Unionsrecht dienten. Soweit die Vertragsverletzungsklage auf eine Verletzung der Gewährleistung effektiven gerichtlichen Rechtsschutzes nach Art. 19 Abs. 1 Unterabs. 2 EUV gestützt ist, sieht Generalanwalt Tanchev einen Vertragsverstoß für gegeben an. Denn die (auch rückwirkende) Herabsetzung des Ruhestandsalters spezifisch für die Mitglieder des Obersten Gerichts im Zusammenspiel mit der Erforderlichkeit einer Zustimmung des Präsidenten der Polnischen Republik für eine Verlängerung der so verkürzten Amtszeit begründeten einen Verstoß gegen die Grundsätze der Unabsetzbarkeit der Richter sowie der richterlichen Unabhängigkeit. Der Generalanwalt stützt sich dabei im Kern auf die Argumentation des Gerichtshofs in seinem Urteil vom 27. Februar 2018 in der Rechtssache C-64/16, Associação Sindical dos Juizes Portugueses. Dort hatte der Gerichthof erstmals festgestellt, dass Art. 19 AEUV die Mitgliedstaaten verpflichte, mittels unabhängiger Gerichte in den vom Unionsrecht erfassten Bereichen einen wirksamen gerichtlichen Rechtsschutz zu gewähren. ${ }^{73}$

68 EuGH, Beschluss der Vizepräsidentin vom 19.10.2018, Rs. C-619/18 R (Kommission/Polen), ECLI:EU:C:2018:852. Mit Beschluss des Präsidenten des EuGH vom 15.11.2018, Rs. C-619/18 (Kommission/Polen), ECLI:EU:C:2018:910, wurde die Rechtssache dem beschleunigten Verfahren unterworfen.

69 Siehe z.B. Handelsblatt v. 17.12.2018, EuGH bestätigt einstweilige Anordnung gegen Polen im Richterstreit, https://www.handelsblatt.com/politik/international/zwangspensionierung-eugh-bestaetigt-einstweilige-anordn ung-gegen-polen-im-richter-streit/23771826.html?ticket=ST-63372-hBY6MceiOaAHqekVTaVq-ap1.

70 Vgl. z.B. https://www.tagesschau.de/ausland/polen-richter-eugh-101.html.

71 EuGH (Große Kammer), Beschluss v. 17.12.2018, Rs. C-619/18 R (Kommission/Polen), ECLI:EU:C:2018:1021.

72 GA Tanchev, Schlussanträge v. 11.4.2019 in der Rs. C-619/18 (Kommission/Polen), ECLI:EU:C:2019:325.

73 EuGH, Urteil v. 27.02.2018, Rs. C-64/16 (Associação Sindical dos Juízes Portugueses), ECLI:EU:C:2018:117, Rn. 40 f. 
Generalanwalt Tanchev geht in seinen Schlussanträgen im Übrigen auch auf die bereits in der Literatur diskutierte Frage ein, ob und inwieweit die vorliegende Vertragsverletzungsklage durch das bezüglich desselben polnischen Gesetzes ausgelöste Rechtsstaatsverfahren nach Art. 7 Abs. 1 EUV gesperrt sein könnte. ${ }^{74}$ Der Generalanwalt weist jedoch darauf hin, dass die Verträge, insbesondere Art. 7 EUV und Art. 258 ff. AEUV selbst, keinen Ausschluss von Vertragsverletzungsverfahren in Materien des Art. 7 EUV vorsehen. Zudem führt er überzeugend aus, dass Natur und Zielsetzung des Art. 7-Verfahrens einerseits und des Vertragsverletzungsverfahrens gem. Art. 258 AEUV andererseits sich essentiell unterscheiden; denn während das Verfahren nach Art. 7 EUV primär politisch geführt wird mit dem Ziel, den betreffenden Mitgliedstaat durch die Androhung bzw. Verhängung von Sanktionen wie dem Stimmrechtsentzug zur Aufgabe einer ,schwerwiegenden und anhaltenden Verletzung" der Werte in Art. 2 EUV zu bringen, handelt es sich beim Vertragsverletzungsverfahren gem. Art. 258 AEUV um ein juristisches Verfahren, an dessen Ende ggf. ein Urteil des Gerichtshofs gem. Art. 260 AEUV mit den sich hieraus ergebenden Rechtsfolgen steht. Generalanwalt Tanchev schließt hieraus, dass die Verfahren nach Art. 7 EUV und Art. 258 AEUV autonom und komplementär nebeneinander stehen. ${ }^{75}$

Der Analyse des Generalanwalts ist im Ergebnis beizupflichten; eine generelle Sperrwirkung des Art. 7 EUV bezüglich Verfahrensgegenständen, die auch die in Art. 2 EUV genannten Werte wie die Rechtsstaatlichkeit berühren, lässt sich nicht überzeugend begründen. Ein Vertragsverletzungsverfahren ist vielmehr dann zulässig, wenn und soweit ein Verstoß gegen EU-Recht vorliegt, der über eine Verletzung der in Art. 2 EUV genannten Werte hinausgeht. Mit anderen Worten dürfte ein unmittelbar auf Art. 2 EUV - etwa das dort niedergelegte Rechtsstaatsprinzip - gestütztes Vertragsverletzungsverfahren unzulässig sein, da Art. 2 EUV selbst weder einen Auftrag zu einer ganz bestimmten rechtlichen Ordnung der Union oder ihrer Mitgliedstaaten statuiert ${ }^{76}$, noch eine eigene Kompetenz der Union zur Durchsetzung der genannten Werte begründet. Soweit ein Verstoß aufgrund darüber hinausgehenden, insoweit einschlägigen bzw. konkretisierenden EU-Rechts - wie im Falle Ungarns und Polens die Richtlinie 2000/78/EG bzw.

74 Siehe zur Diskussion dieser Frage in der Literatur etwa Editorial Comments, Safeguarding EU values in the Member States - Is something finally happening?, CMLRev. Vol. 52 (2015), S. 619, 626. Für ein Nebeneinander beider Verfahrensarten z.B. S. Kadelbach, Verfassungskrisen in der Europäischen Union, in: ders. (Hrsg.), Verfassungskrisen in der Europäischen Union, 2018, S. 9, 18 f. (mit Hinweis auf die unterschiedliche Zweckrichtung und Rechtsfolgen der jeweiligen Verfahren).

75 GA Tanchev, Schlussanträge v. 11.4.2019 in der Rs. C-619/18 (Kommission/Polen), ECLI:EU:2019:325, Rn. 50.

76 J. Schwarze/N. Wunderlich, in Schwarze/Becker/Hatje/Schoo (Hrsg.), EU-Kommentar, 4. Aufl. 2019, Art. 2 EUV Rn. 2, 3. Weitergehend, ein auf Art. 2 EUV gestütztes Vertragsverletzungsverfahren, wohl für zulässig haltend, A. Weber, Europäisches Rechtsdenken in der Krise?, DÖV 2017, S. 741, 748. 
Art. 19 EUV $^{77}$ - in Rede steht, erscheint die Einleitung eines Vertragsverletzungsverfahrens jedoch aus Kompetenzgesichtspunkten rechtlich zulässig. ${ }^{78}$

Der Gerichtshof folgte in seinem in der Großen Kammer gefassten Urteil vom 24. Juni 2019 im Ergebnis den Schlussanträgen von Generalanwalt Tanchev. Er stellte einen Verstoß gegen Art. 19 EUV fest und hob dabei hervor, dass der Grundsatz der Unabsetzbarkeit von Richtern unmittelbar mit dem Prinzip von deren Unabhängigkeit (Art. 19 Abs. 1 Unterabs. 2 EUV) verknüpft sei ${ }^{79}$.

\section{Zusammenfassung/Schluss}

Zusammenfassend lässt sich festhalten, dass gerade in den letzten Jahren um das geltende Recht in der EU und seine Auslegung stark gerungen wird und eine Reihe mitgliedstaatlicher Maßnahmen bezüglich ihrer Vereinbarkeit mit Unionsrecht kontrovers diskutiert werden. Aufgrund der Finanz- und Wirtschaftskrise sowie der Migrationskrise hat dieses Ringen um deren rechtliche Bewältigung eine besondere Dimension erhalten. Im Zusammenhang mit beiden Krisensituationen wurde zu Recht darauf hingewiesen, dass das bestehende Recht keine passgenauen Lösungen für die plötzlichen Umbrüche bereithielt. Die Mitgliedstaaten ebenso wie die EU und ihre Institutionen - waren daher gezwungen, mit Hilfe des bestehenden Rechtsinstrumentariums dieser neuen Situation Herr zu werden, ohne dass insoweit Leitlinien etwa durch die Rechtsprechung bestanden hätten. Entscheidend für die Frage, ob die Mitgliedstaaten in einer solchen Situation tatsächlich Recht „missachtet“ haben, ist dabei, ob sie sich letztlich einem Kontrollsystem und insbesondere einer letztverbindlichen Gerichtsentscheidung unterworfen haben.

Wie sich aus der hier vorgelegten Bestandsaufnahme ergibt, die selbstverständlich keinen Anspruch auf Vollständigkeit erhebt, dürfte eine solche grundsätzliche Rechtstreue ganz überwiegend anzunehmen sein, selbst wenn Diskussionen über das Recht und die Rechtsauslegung nicht abreißen. Eine Erschütterung der Rechtsgemeinschaft, deren Kern in der Unterwerfung unter eine rechtliche und

77 Siehe zur Frage, inwieweit Art. 19 EUV Vorgaben für die Ausgestaltung des nationalen Rechtsschutzes enthält, z.B. EuGH, Urt. v. 27.2.2018, Rs. C-64/16 (ASJP/Tribunal de Contas), ECLI:EU:C:2018:117 (zur Frage der Unabhängigkeit des Tribunal de Contas). Zur Frage der Unabhängigkeit der rumänischen Justiz (insbes. Sonderstaatsanwaltschaft für Ermittlungen gegen Richter und Staatsanwälte) siehe verb. Rs. C-83/19, C-127/19 und C-195/19. Siehe zu diesem Fragenkreis auch den Vorlagebeschluss VG Wiesbaden vom 28.3.19 (Az. 6 K 1016/15) zur Frage der Unabhängigkeit dieses Gerichts (Rs. C-272/19). Mit Blick auf die Unabhängigkeit der Justiz in Polen siehe auch Vorlagen der polnischen Gerichte selbst: Rs. C-522/18 (ABl. EU 2018 C 427/8); Rs. C-537/18 (ABl. EU 2018 C 427/11); verb. Rs. C-585/18, C-624/18, C-625/18 (ABl. EU 2019 C 44/10); verb. Rs. C-558/18 (Abl. EU 2019 C 44/8), C-563/18 (ABl. EU 2019 C 44/9) (Vorlage der Bezirksgerichte Łódź und Warschau betr. disziplinarische Kontrolle von Richtern).

78 Ein weiteres Vertragsverletzungsverfahren, das die Kommission am 3.4.2019 gegen Polen aufgrund einer neuen Disziplinarregelung für Richter eingeleitet hat, ist ebenfalls auf Art. 19 EUV und Art. 47 GRC gestützt; siehe Pressemitteilung der Europäischen Kommission v. 3.4.2019, http://europa.eu/rapid/press-release_IP-191957_de.htm. Siehe zudem das beim EuGH anhängige Vertragsverletzungsverfahren C-192/18 (Kommission/ Polen) (ABl. EU 2018 C 182/14) und hierzu GA Tanchev, Schlussanträge v. 20.6.19 in der Rs. C-192/18 (Kommission/Polen), ECLI:EU:C:2019:529.

79 EuGH, Urt. v. 24.06.2019, Rs. C-619/18 (Kommission/Polen), ECLI:EU:C:2019:531, Rn. 96. 
insbesondere gerichtliche Kontrolle liegt, läge dann vor, wenn es zu systematischen Rechtsverletzungen und einer grundsätzlichen Verweigerungshaltung gegenüber den Rechtsvorschriften der EU und vor allem den Urteilen des Gerichtshofs käme. Auch wenn einzelne Entwicklungen erhebliche Bedenken aufwerfen, erscheint unter Zugrundelegung dieses Maßstabs die Rechtsgemeinschaft als Ganze jedoch (noch) nicht unmittelbar gefährdet. 